\title{
Using Hyperfine Electron Paramagnetic Resonance Spectroscopy to Define the Proton-Coupled Electron Transfer Reaction at Fe-S Cluster N2 in Respiratory Complex I
}

\author{
Nolwenn Le Breton, ${ }^{\dagger}$ John J. Wright, ${ }^{\dagger}$ Andrew J. Y. Jones, ${ }^{\ddagger}$ Enrico Salvadori, ${ }^{\dagger}$, Hannah R. Bridges, ${ }^{\ddagger}$ \\ Judy Hirst, ${ }^{\ddagger 0}$ and Maxie M. Roessler*, ${ }^{*} \dagger$ \\ ${ }^{\dagger}$ School of Biological and Chemical Sciences, Queen Mary University of London, Mile End Road, London, E1 4NS, United Kingdom \\ ${ }^{\ddagger}$ Medical Research Council Mitochondrial Biology Unit, University of Cambridge, Wellcome Trust/MRC Building, Cambridge \\ Biomedical Campus, Hills Road, Cambridge CB2 0XY, United Kingdom \\ ${ }^{\S}$ London Centre for Nanotechnology, University College London, 17-19 Gordon Street, London WC1H 0AH, United Kingdom
}

Supporting Information

ABSTRACT: Energy-transducing respiratory complex I (NADH:ubiquinone oxidoreductase) is one of the largest and most complicated enzymes in mammalian cells. Here, we used hyperfine electron paramagnetic resonance (EPR) spectroscopic methods, combined with site-directed mutagenesis, to determine the mechanism of a single proton-coupled electron transfer reaction at one of eight iron-sulfur clusters in complex I, [4Fe-4S] cluster N2. N2 is the terminal cluster of the enzyme's intramolecular electron-transfer chain and the electron donor to ubiquinone. Because of its position and $\mathrm{pH}$-dependent reduction potential, $\mathrm{N} 2$ has long been considered a candidate for the elusive "energy-coupling" site in complex I at which energy generated by the redox reaction is used to initiate proton translocation. Here, we used hyperfine sublevel correlation (HYSCORE) spectroscopy, including relaxation-filtered hyperfine and single-matched resonance transfer (SMART) HYSCORE, to detect two weakly coupled exchangeable protons near N2. We assign the larger coupling with $A\left({ }^{1} \mathrm{H}\right)=[-3.0,-3.0,8.7] \mathrm{MHz}$ to the exchangeable proton of a conserved histidine and conclude that the histidine is hydrogen-bonded to $\mathrm{N} 2$, tuning its reduction potential. The histidine protonation state responds to the cluster oxidation state, but the two are not coupled sufficiently strongly to catalyze a stoichiometric and efficient energy transduction reaction. We thus exclude cluster N2, despite its proton-coupled electron transfer chemistry, as the energy-coupling site in complex I. Our work demonstrates the capability of pulse EPR methods for providing detailed information on the properties of individual protons in even the most challenging of energy-converting enzymes.

\section{INTRODUCTION}

Mitochondrial complex I (NADH:ubiquinone oxidoreductase) is a redox-coupled proton pumping enzyme that is essential for respiration. By catalyzing $\mathrm{NADH}$ oxidation and quinone reduction, it contributes to the proton motive force across the mitochondrial inner membrane that drives the synthesis of ATP. Knowledge of the mechanism of complex I is not only of fundamental importance for understanding proton-coupled electron transfer (PCET) reactions but also required to unravel the molecular origins of a diverse range of neuromuscular and neurodegenerative diseases. ${ }^{1,2}$ Complex I is the most enigmatic of the proton-translocating respiratory enzymes, and the mechanism of its redox-coupled proton transfer chemistry is currently unknown. ${ }^{3}$

Recently, much progress has been made in structural knowledge of complex I. The structure of the entire Thermus thermophilus complex ${ }^{4}$ was followed by structures of its yeast and mammalian homologues, which are almost twice the size of the bacterial enzyme. ${ }^{5-8}$ The structures of the catalytically active core subunits are closely conserved in all cases, consistent with a conserved mechanism of catalysis. They show that the two substrate-binding sites in complex I, the flavin-containing $\mathrm{NADH}$ binding site and the quinone binding site, are separated by almost $70 \AA$ and connected by an electron-transfer relay of seven $\mathrm{Fe}-\mathrm{S}$ clusters to shuttle electrons between them (Figure 1). Near-linear $\mathrm{Fe}-\mathrm{S}$ relays of this kind are common in metalloenzymes, ${ }^{9-12}$ but the relay in complex I is exceptionally long and its pattern of alternating high and low potential centers $^{13}$ likely contributes to intramolecular electron transfer being at least an order of magnitude faster than enzyme turnover. ${ }^{14,15}$ Intriguingly, $\mathrm{Fe}-\mathrm{S}$ cluster relays are increasingly being found to have important roles beyond electron transfer. The cluster furthest from the active site in $[\mathrm{NiFe}]$-hydrogenases exerts a strong influence on the kinetics of catalysis, ${ }^{16,17}$ whereas the cluster adjacent to the active site plays a crucial role in protecting it against $\mathrm{O}_{2}{ }^{18,19}$ The unusual [4Fe-3S] hydrogenase cluster is evolutionarily related to $[4 \mathrm{Fe}-4 \mathrm{~S}]$ cluster

Received: September 2, 2017

Published: October 17, 2017 


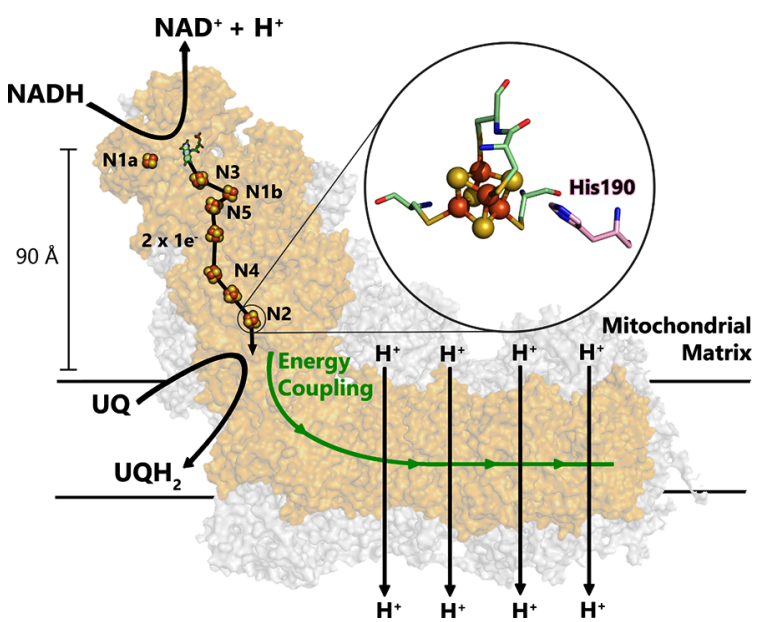

Figure 1. Reactions catalyzed by respiratory complex I and the environment of cluster $\mathrm{N} 2$. The $\mathrm{Fe}-\mathrm{S}$ clusters are labeled according to their EPR signals. Core subunits are in beige, and supernumerary subunits are in gray. The inset shows $\mathrm{N} 2$ rotated with respect to the chain with the conserved histidine in the second coordination sphere; residues from the $49 \mathrm{kDa}$ and PSST subunits are shown in green and pink, respectively. PDB ID: 5LDW (bovine complex I).

$\mathrm{N} 2{ }^{20}$ the terminal cluster in complex I's intramolecular electron transfer chain (Figure 1), which has been suggested to synchronize electron and proton transfer events. ${ }^{15}$

As the immediate electron donor to ubiquinone, cluster N2 has long been considered a candidate "energy-coupling" site in complex I, i.e., the site at which redox catalysis initiates proton translocation across the membrane. ${ }^{21,22}$ As there is no evidence to suggest that any upstream electron transfers (from $\mathrm{NADH}$ toward N2) are coupled to proton pumping, the alternative candidate is the quinone/quinol-binding site. N2 and the bound quinone headgroup are known to be in close proximity: densities observed in cocrystallization experiments with a shortchain analogue suggested that the quinone headgroup binds within $12 \AA$ of cluster $\mathrm{N}^{4}$ (within electron-transfer distance), consistent with earlier data from electron paramagnetic resonance (EPR) spectroscopy. ${ }^{23}$

The reduction potential of $\mathrm{N} 2$ has long been known to be $\mathrm{pH}$ dependent ${ }^{22}$ suggesting it may carry out a crucial PCET reaction during complex I catalysis (we use the term PCET to describe reactions in which the occupancy of a protonatable site changes in response to cluster reduction). However, a lack of consensus on the extent of the $\mathrm{pH}$ dependence, particularly for the well-studied mammalian complex, ${ }^{3}$ has hindered mechanistic interpretations. Importantly, a strongly coupled PCET reaction, as required for an energy-coupling site, would exhibit a $\mathrm{pH}$ dependence of $-60 \mathrm{mV}$ per $\mathrm{pH}$ unit with a substantial separation of the $\mathrm{p} K$ values for the oxidized and reduced states to ensure stoichiometric proton uptake upon reduction. A -60 $\mathrm{mV}$ dependence was reported for the mammalian (Bos taurus) complex, but in complex I from the aerobic yeast Yarrowia lipolytica, it was found to be substantially less $(-36 \mathrm{mV} / \mathrm{pH})$ and to be abolished by a single point mutation (H226M in the $49 \mathrm{kDa}$ subunit). ${ }^{24}$ Density for His226 was not observed in the structure of $Y$. lipolytica complex $\mathrm{I}^{7}$ but its homologous residue in the bovine enzyme His 190 was observed in close proximity of cluster N2 (Figure 1).

Investigating the role of protons in large metalloenzymes such as complex I by observing them directly (rather than indirectly through $\mathrm{pH}$-dependent potentiometric titrations) is a challenging task. For complex I, available structures lack the resolution required to define the protonation states of amino acids, or the presence of water molecules, in the vicinity of cluster N2. By examining the hyperfine interactions between the electron spin on the cluster and nearby nuclear spins (e.g., ${ }^{1} \mathrm{H}$ ), pulse EPR spectroscopy is ideally suited to provide this missing information. However, although numerous continuouswave EPR spectroscopic studies have determined the nature and redox properties of the EPR-visible clusters in complex $\mathrm{I}^{22,25-29}$ few pulse EPR studies have been reported. Relaxationfiltered hyperfine (REFINE) spectroscopy predicted the absence of a nitrogen nucleus in the primary coordination sphere of N2, ${ }^{30,31}$ as later confirmed structurally (Figure 1), and double electron-electron resonance spectroscopy enabled a definitive assignment of the spectroscopically observed clusters to the structurally defined clusters through distance measurements. $^{13}$

Here, we investigate the existence of exchangeable protons in the environment of $\mathrm{N} 2$ both indirectly, using $\mathrm{pH}$-dependent reduction potential measurements, and directly and selectively using pulse EPR spectroscopy. First, we define the $\mathrm{pH}$ dependent reduction potential of $\mathrm{N} 2$ in the mammalian complex using small-volume potentiometric titrations ${ }^{32}$ under tightly defined conditions: we develop a widely applicable buffer system over a wide $\mathrm{pH}$ range that accounts for temperature-dependent changes in $\mathrm{pH}$ during sample preparation that could otherwise lead to misleading results. ${ }^{32,33}$ Then, we apply pulse EPR measurements to detect exchangeable protons in the vicinity of the reduced N2 cluster. We reveal the presence of a hydrogen bond to a conserved histidine in the second coordination sphere of the cluster and confirm the protonation state of the histidine as the origin of the $\mathrm{pH}$ dependent reduction potential. By defining the PCET reaction of cluster N2, we show that its behavior is not consistent with it being the energy-coupling reaction in complex I, directing the focus of future work to the coupled chemistry of quinone reduction. Thus, we demonstrate the capability of pulse EPR methods for interrogating mechanistic hypotheses involving the properties of individual protons in large and experimentally challenging energy-transducing complexes.

\section{RESULTS AND DISCUSSION}

pH Dependence of the N2 Reduction Potential. To establish unambiguously the $\mathrm{pH}$ dependence of the reduction potential of $\mathrm{N} 2$ in mammalian complex I, we carried out a series of EPR-monitored small-volume redox titrations, at $\mathrm{pH}$ values from 5 to 9, on the bovine enzyme (Supplementary Figure 1). The data are summarized in Figure 2. Temperatureindependent $\mathrm{pH}$ buffer systems, the $\mathrm{pH}$ values of which are unchanged from room to cryogenic temperatures (Supplementary Figure 2), were used to obtain the data points at $\mathrm{pH}$ 6.0, 7.0, 8.0, and 9.0. We note that substantial temperaturedependent $\mathrm{pH}$ changes of commonly used buffers have been known for four decades, ${ }^{33}$ but their impact on measurements such as these is often ignored; the systems devised here (Supplementary Table 1) are suitable for wider applications. Furthermore, because bovine complex I proved to be unstable at $\mathrm{pH}<6$ for the $\sim 3 \mathrm{~h}$ required for a titration, we obtained the data point at $\mathrm{pH} 5.0$ by exploiting the dramatic $\mathrm{pH}$ change that occurs in certain buffers upon freezing and could thus obtain a reliable measurement under this challenging condition (see Experimental Section). A data point recorded at $\mathrm{pH} 7.4$ (see 


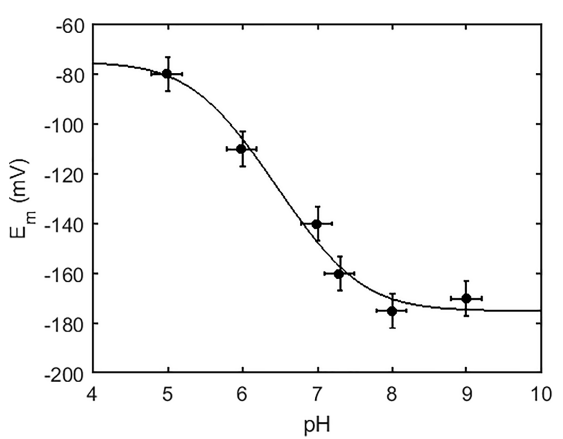

Figure 2. $\mathrm{pH}$-dependent reduction potential of $\mathrm{Fe}-\mathrm{S}$ cluster $\mathrm{N} 2$ in bovine complex I. Data points resulting from EPR-monitored potentiometric titrations (see Experimental Methods and Supplementary Figure 1) were fitted with a single proton with $\mathrm{p} K_{\mathrm{ox}}=5.6 \pm$ $0.2, \mathrm{p} K_{\text {red }}=7.4 \pm 0.2, E_{\text {acid }}=-75 \pm 5 \mathrm{mV}$, and $E_{\text {alk }}=-175 \pm 5 \mathrm{mV}$ (solid line).

Figure 2) using the same approach confirmed the validity of this alternative strategy.

The solid line in Figure 2 represents the best fit to the data for a single protonated group and has a maximum slope of -40 $\mathrm{mV}$ per $\mathrm{pH}$ unit. The $\mathrm{pK}$ values for the protonable group are $\mathrm{p} K_{\mathrm{ox}}=5.6 \pm 0.2$ and $\mathrm{p} K_{\mathrm{red}}=7.4 \pm 0.2$, and the reduction potentials of $\mathrm{N} 2$ are $E_{\text {acid }}=-75 \pm 5 \mathrm{mV}$ and $E_{\text {alk }}=-175 \pm 5$ $\mathrm{mV}$ in the protonated and deprotonated forms, respectively. In principle, multiple more weakly coupled protons could also give rise to the same $\mathrm{pH}$ dependence, as was found to be case for Escherichia coli cytochrome $b-562 .{ }^{34}$ However, the transfer of a single proton together with two electrons (to give a $\mathrm{pH}$ dependence of $-30 \mathrm{mV} / \mathrm{pH}$ ) can be excluded because $\mathrm{N} 2$ exhibits only two oxidation states $\left([4 \mathrm{Fe}-4 \mathrm{~S}]^{2+/+}\right) .^{3}$

The $\mathrm{pH}$ dependence determined does not match the data reported previously for bovine complex I in submitochondrial particles or mitochondria, which exhibited slopes of $-60 \mathrm{mV} /$ $\mathrm{pH}$ unit, from approximately $\mathrm{pH} 6.2$ to $8.7 .^{22}$ However, our data are consistent with data on complex I in mitochondrial membranes from $Y$. lipolytica conducted over a wider $\mathrm{pH}$ range ( $\mathrm{pH}$ 5.0-9.0). ${ }^{24}$ Uncertainties in the buffer $\mathrm{pH}$ values in earlier studies offer a possible explanation for the disagreement in the $\mathrm{pH}$ dependencies obtained. The moderate $\mathrm{pH}$ dependence of the $\mathrm{N} 2$ reduction potential, observed now in both species, suggests that the PCET reaction occurring at N2 is only weakly coupled and thus argues against it being the mechanistic coupling point in complex I. However, titration data can only provide indirect information on the existence of exchangeable proton(s). Thus, to investigate the proton environment of cluster N2 directly and its interactions with exchangeable protons in detail, we turned to hyperfine spectroscopic techniques.

The Exchangeable Proton Environment of N2 (Bovine Complex I). To enable selective investigation of the proton environment of cluster N2 without interference from the many other $\mathrm{Fe}-\mathrm{S}$ clusters (Figure 1), we chose samples set at reduction potentials at which $\mathrm{N} 2$ is the sole paramagnetic species present (see Experimental Methods and Supplementary Figure 3). Structural data on bovine complex $I^{6}$ suggests that there are approximately 40 protons within $5 \AA$ of $\mathrm{N} 2$, so assignment of all the proton couplings observed by hyperfine spectroscopy is unrealistic, especially given the limited resolution of available structures. We thus focused on the exchangeable protons, using deuterium exchange, and by investigating the $\mathrm{pH}$ dependence of hyperfine couplings.

In agreement with the data in Figure 2, only weakly coupled protons were observed in hyperfine sublevel correlation (HYSCORE) spectra. To observe differences in the weakly coupled protons clearly (see Supplementary Figures 4 and 5 for primary data), we employed previously described HYSCORE subtraction procedures. ${ }^{35,36}$ The HYSCORE difference spectra obtained for pH 6.0 vs. pD 6.0 (H vs. D) and for $\mathrm{pH} 6.0$ vs. $\mathrm{pH}$ 9.0 (low $\mathrm{pH}$ vs. high $\mathrm{pH}$ ) are shown in Figure $3 \mathrm{~A}$ and $\mathrm{B}$ (where

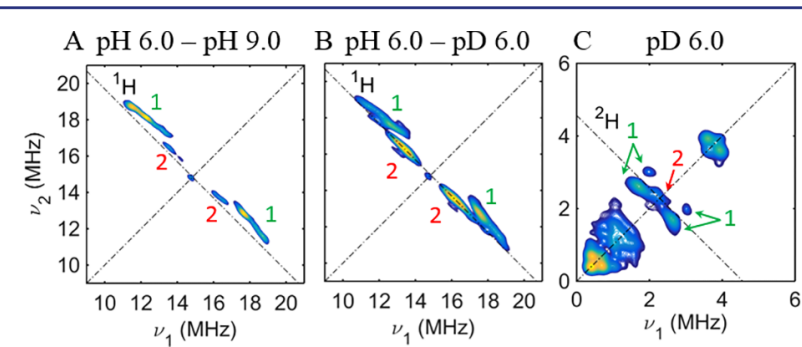

Figure 3. HYSCORE spectra showing the exchangeable protons in the vicinity of $\mathrm{N} 2$ in bovine complex I. (A) [pH 6.0-pH 9.0] and (B) $[\mathrm{pH}$ 6.0-pD 6.0] difference spectra. (C) HYSCORE spectrum showing the deuterium coupling only present in the deuterated samples $(\mathrm{pD} 6.0$ ). Spectra were recorded at $10 \mathrm{~K}$ at $g_{\perp}$, time increment $16 \mathrm{~ns}, \tau=116 \mathrm{~ns}$ (A and B), or time increment $24 \mathrm{~ns}, \tau=420 \mathrm{~ns}(\mathrm{C})$. See Experimental Methods for sample preparation, further measurement conditions, and HYSCORE subtraction procedures.

$\mathrm{pH} 6.0$ is $1.4 \mathrm{pH}$ units below $\left.\mathrm{p} K_{\text {red }}\right)$. Both spectra reveal two sets of cross peaks centered at approximately $[17.8,12.5]$ and $[16.4,13.4] \mathrm{MHz}$. HYSCORE difference spectra at additional field positions, required because the microwave pulses cannot excite the entire broad $\mathrm{Fe}-\mathrm{S}$ cluster EPR spectrum (resulting in orientation selection), also exhibit two sets of cross peaks (Supplementary Figure 6). As expected from the exchangeable proton couplings revealed in the difference spectra (labeled 1 and 2), the $\mathrm{pD} 6.0$ sample gave rise to small additional couplings $(<2 \mathrm{MHz})$ in the ${ }^{2} \mathrm{H}$ region (Figure $\left.3 \mathrm{C}\right)$. We could not detect significant differences in the nitrogen region in low $\mathrm{pH}$ vs high $\mathrm{pH}$ spectra (Supplementary Figure 4A and B) nor in the ${ }^{14} \mathrm{~N}$ difference spectra (not shown). A comparison of the nitrogen region in the $\mathrm{H}$ vs $\mathrm{D}$ spectra is not feasible because the cross-peaks resulting from ${ }^{14} \mathrm{~N}$ and ${ }^{2} \mathrm{H}$ (both quadrupolar nuclei with $I=1$ ) overlap. The unaltered ${ }^{14} \mathrm{~N}$ region suggests that there are no substantial changes in the protonation/ bonding state of any ${ }^{14} \mathrm{~N}$ nucleus in the immediate vicinity of N2.

Using the method developed by Dikanov and co-workers to analyze orientation-selected HYSCORE spectra ${ }^{36,37}$ as a starting point to determine the hyperfine coupling parameters (Supplementary Table 2), we found that our data are well described by two weakly coupled protons, $\mathrm{H} 1$ and $\mathrm{H} 2$ (Supplementary Figures 8 and $9 \mathrm{~A}-\mathrm{C}$ ) with the parameters in Table 1 (see also Supplementary Table 3). The deuterium coupling (Figure 3C) could be simulated well (Supplementary Figure 9D) using the parameters for $\mathrm{H} 1$ and accounting for the smaller gyromagnetic ratio of deuterium and including a quadrupole coupling (Supplementary Table 3). The S*H hydrogen bond was estimated from the quadrupole coupling to be $1.77 \pm 0.29 \AA$, which compares favorably with donoracceptor distances from structural data (see Table 1 and Supplementary Table 4). The hyperfine coupling of D2 is 
Table 1. ${ }^{1} \mathrm{H}$ Hyperfine Coupling Parameters for $\mathrm{H} 1$ and $\mathrm{H} 2$ Compared to Selected Parameters Reported in the Literature ${ }^{a}$

\begin{tabular}{lcccc} 
& & & \multicolumn{2}{c}{ H-bond length $(\AA)$} \\
\cline { 3 - 4 } H1 (complex I) & $A_{\text {iso }}(\mathrm{MHz})$ & $T(\mathrm{MHz})$ & structure $(\mathrm{D} \cdots \cdot \mathrm{A})$ & quadrupole $(\mathrm{H} \cdots \cdot \mathrm{A})$ \\
$\mathrm{H} 2$ (complex I) & 0.9 & 3.9 & $2.9-4.0^{b}$ & 1.77 \\
Leu-[2Fe-2S] (Rieske protein) $)^{36}$ & 0 & 3.2 & $3.4^{c}$ & 1.62 \\
His-semiquinone (nitrate reductase) & 39 & 4.94 & & \\
His/Asp-semiquinone (complex III) & -1.69 & 5.67 & 2.5 & \\
& 1.8 & -4.2 & 2.7 & -5.5
\end{tabular}

${ }^{a}$ See Supplementary Table 3 for the complete set of simulation parameters for $\mathrm{H} 1$ and $\mathrm{H} 2$. Note that $A=A_{\text {iso }}+(-T,-T,+2 T)$. H-bond lengths refer to the donor-acceptor $(\mathrm{D} \cdots \mathrm{A})$ distances from structural data or to hydrogen-acceptor $(\mathrm{H} \cdots \mathrm{A})$ distances $(r)$ calculated from the quadrupole coupling using the equation (developed for $\mathrm{N}-\mathrm{H} \cdots \mathrm{O}$ bonds) $\frac{e^{2} q Q}{h}=a-b /\left(r_{\mathrm{H} \cdots \mathrm{A}}^{3}\right)[\mathrm{kHz}]$, where $a=319 \mathrm{kHz}$ and $b=607 \mathrm{kHz} \AA^{3} .38 b_{\mathrm{Th}} \mathrm{D} \cdots \mathrm{A}$ distance range is based on currently available structural data (see Supplementary Table 4). ${ }^{c} \mathrm{~S}_{\mathrm{FeS}_{\mathrm{Fe}} \cdots \mathrm{N}_{\mathrm{Leu} 132}}$ distance from structure (PDB ID: 2NUK).

expected to be very small $(<0.5 \mathrm{MHz})$ and, with the $\tau$ value of the experiment chosen to place a blind spot on the deuterium Larmor frequency $(2.3 \mathrm{MHz}$ at $349.5 \mathrm{mT})$, is not clearly visible (Figure 3C).

Figure 4 shows that exchangeable proton couplings can also be detected directly at $\mathrm{pH} 6.0$ using single-matched resonance

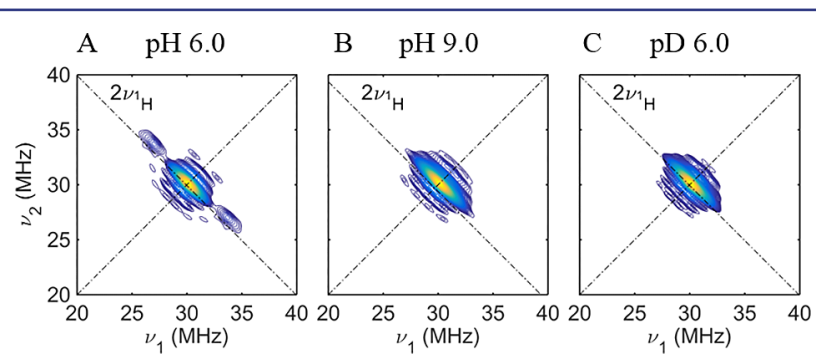

Figure 4. SMART-HYSCORE spectra of N2 in bovine complex I at pH 6.0 (A), pH 9.0 (B), and pD 6.0 (C). Spectra were recorded at 10 $\mathrm{K}$ at $g_{\perp}$, time increment of $8 \mathrm{~ns}, \tau=96 \mathrm{~ns}$. See Supplementary Figure 7 for full spectrum of (A) and Experimental Methods for sample preparation and measurement conditions.

transfer (SMART) HYSCORE. In this experiment, ${ }^{41}$ which unlike the HYSCORE experiment does not suffer from blind spots, well-resolved double-quantum cross-peaks that correlate two different protons are visible (Figure 4A) along the antidiagonal at twice the proton Larmor frequency $(\sim 30$ $\mathrm{MHz}$ ). These peaks are not visible in the $\mathrm{pD} 6.0$ or $\mathrm{pH} 9.0$ samples (Figure 4B and C). The SMART HYSCORE $\mathrm{pH} 6.0$ spectrum is described well by the hyperfine coupling parameters for $\mathrm{H} 1$ and $\mathrm{H} 2$ given in Table 1 (Supplementary Figure 9E). One of the two protons giving rise to the combination peaks along $2 \nu_{1 \mathrm{H}}$ in Figure $4 \mathrm{~A}$ must be $\mathrm{H} 1$ to give rise to the significant splitting observed. A nonexchangeable proton with similar parameters to $\mathrm{H} 1$ can be excluded because the combination peaks should then also be present in the $\mathrm{pD}$ 6.0 and $\mathrm{pH} 9.0$ spectra. However, the origin of the second proton giving rise to the combination peak in Figure 4A is less clear because reasonable fits to the experimental data could also be obtained using alternative (weak-coupling) parameters for $\mathrm{H} 2$. Given that nonexchangeable protons with similar-sized couplings to $\mathrm{H} 1$ are present in the HYSCORE spectra (Supplementary Figure 5), it is remarkable that these do not give rise to combination peaks centered around $\left(2 \nu^{1}{ }_{\mathrm{H}}, 2 \nu_{\mathrm{H}}^{1}\right)$. We found that the combination peaks observed in the $\mathrm{pH} 6.0$ SMART HYSCORE spectrum are highly sensitive to the angle between the hyperfine tensors of $\mathrm{H} 1$ and $\mathrm{H} 2$ (Supplementary
Figure 10). We therefore conclude that it is the orientation of the $\mathrm{H} 1$ hyperfine tensor that enabled us to observe the combination peak resulting from the $\mathrm{H} 1$ and $\mathrm{H} 2$ transitions directly using SMART HYSCORE. We also attempted Q-band Mims electron nuclear double resonance (ENDOR) measurements (data not shown) but found differences in the proton couplings were better resolved using HYSCORE techniques given the relatively low concentrations of our samples and the small sample sizes.

Thus, the couplings from two exchangeable protons, $\mathrm{H} 1$ and $\mathrm{H} 2$, are present at $\mathrm{pH} 6.0\left(<\mathrm{p} K_{\text {red }}\right)$ but absent at $\mathrm{pH} 9.0$ $\left(>\mathrm{p} K_{\text {red }}\right)$ or following deuterium exchange: the protonable groups that produce these couplings must have $\mathrm{p} K_{\mathrm{a}}$ values between 6 and 9. Previously, the $\mathrm{pH}$ dependence of the reduction potential of cluster N2 in Y. lipolytica was found to be ablated by the mutation of a nearby highly conserved histidine residue (His226 in Y. lipolytica, corresponding to His190 in B. taurus, see Figure 1). Thus, it is likely that either $\mathrm{H} 1$ or $\mathrm{H} 2$ arise from His190. To assist with the assignment of $\mathrm{H} 1$ and $\mathrm{H} 2$, we thus compared the proton environment of cluster N2 in the wild-type and H226M variant of complex I from Y. lipolytica.

Assignment of Exchangeable Proton $\mathrm{H} 1$ Using $Y$. lipolytica Complex I. When His226 in Y. lipolytica complex I was mutated to methionine, a residue with no exchangeable protons, the reduction potential of $\mathrm{N} 2$ decreased to a $\mathrm{pH}$ independent value of $-216 \mathrm{mV} .^{24}$ If His226 gives rise to either $\mathrm{H} 1$ or $\mathrm{H} 2$, then one of the proton couplings described above will be absent in the $\mathrm{H} 226 \mathrm{M}$ variant. In $\mathrm{H} 226 \mathrm{M}$, selective reduction of $\mathrm{N} 2$ is not possible as its reduction potential is close to that of the other EPR-visible $\mathrm{Fe}-\mathrm{S}$ clusters. We thus took advantage of the different relaxation times of $\mathrm{N} 2$, relative to that of the $[2 \mathrm{Fe}-2 \mathrm{~S}]$ cluster $\mathrm{N} 1 \mathrm{~b}$, which is also visible at the maximum acceptable measurement temperature for HYSCORE spectra with good signal-to-noise $(14 \mathrm{~K})$. We thus employed relaxation filters (REFINE spectroscopy) $)^{30}$ to measure the proton hyperfine couplings to N2 selectively (Supplementary Figure 11). The resulting REFINE-HYSCORE difference spectra are shown in Figure 5.

The [wild-type - H226M] difference spectrum ( $\mathrm{pH}$ 6.5) clearly reveals a single set of proton cross-peaks (Figure 5A) with hyperfine coupling parameters that match $\mathrm{H} 1$ (Table 1). In contrast, the $[\mathrm{pH} 6.5-\mathrm{pD} 6.5]$ difference spectrum of the wild-type shows two sets of cross peaks (Figure 5B) that are essentially identical to bovine $\mathrm{H} 1$ and $\mathrm{H} 2$ (Figure 3B). Therefore, we conclude that $\mathrm{H} 1$ arises from the exchangeable proton of His226 in Y. lipolytica complex I and His190 in bovine complex I. As for bovine complex I, we could not detect 


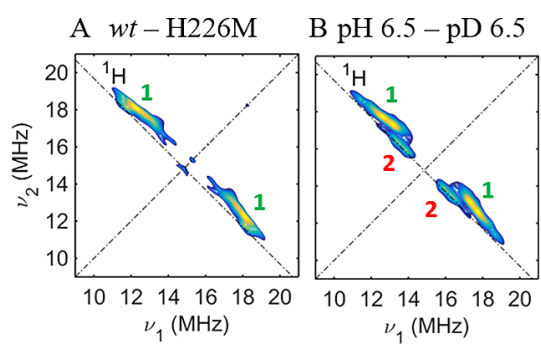

Figure 5. HYSCORE spectra showing the exchangeable protons in the vicinity of $\mathrm{N} 2$ in wild-type and $\mathrm{H} 226 \mathrm{M} \mathrm{Y}$. lipolytica complex I. (A) [Wild-type $-\mathrm{H} 226 \mathrm{M}$ ] symmetrized difference spectrum $(\mathrm{pH} 6.5)$ and (B) wild-type $[\mathrm{pH} 6.0-\mathrm{pD} 6.0]$ symmetrized difference spectrum. Spectra were recorded at $14 \mathrm{~K}$ at $g_{\perp}$, time increment $16 \mathrm{~ns}, \tau=116 \mathrm{~ns}$, and filtering time $T_{\mathrm{f}}=62 \mu \mathrm{s}$. See Experimental Methods for sample preparation, further measurement conditions, and HYSCORE subtraction and symmetrization procedures.

significant differences in the ${ }^{14} \mathrm{~N}$ couplings in $Y$. lipolytica complex I difference spectra.

$\mathrm{H} 1$ is Hydrogen-Bonded to Cluster N2. The isotropic hyperfine coupling $\left(A_{\text {iso }}\right)$ of $\mathrm{H} 1$ shows that a substantial amount of electron spin density is present on the exchangeable proton of His 190/226 (Table 1). It was not possible to fit the data satisfactorily with $A_{\text {iso }}$ set to zero. His $190 / 226$ is present in the second coordination sphere of $\mathrm{N} 2$, so there are no throughbond interactions to explain the nonzero $A_{\text {iso }}$. However, the anisotropic through-space interaction $(T=3.9 \mathrm{MHz})$ is also relatively large, suggesting a close association between the proton and the reduced cluster. We thus propose that His190/ 226 forms a hydrogen bond to the reduced [4Fe-4S] N2 cluster. Hydrogen bonds have long been known to play an important role in modulating the reduction potentials of $\mathrm{Fe}-\mathrm{S}$ clusters, and a recent ultrahigh resolution $(0.48 \AA)$ structure of a high-potential iron-sulfur protein from Thermochromatium tepidum has revealed the extensive hydrogen-bonding network around the $[4 \mathrm{Fe}-4 \mathrm{~S}]$ cluster. $^{42,43}$ However, to the best of our knowledge, only one set of hyperfine parameters for a proton hydrogen-bonded to a $\mathrm{Fe}-\mathrm{S}$ cluster (the $[2 \mathrm{Fe}-2 \mathrm{~S}]$ cluster in a Rieske protein, Table 1) has been reported. ${ }^{36}\left|A_{\text {iso }}\right|$ and $|T|$ for this leucine proton are comparable to the hyperfine coupling parameters for $\mathrm{H} 1$. The anisotropy of $\mathrm{H} 1$ also compares favorably with values reported for His residues hydrogen bonded to semiquinones (Table 1). For cluster N2, hydrogen bonding could involve either the bridging sulfides of the $\mathrm{Fe}-\mathrm{S}$ cubane or the sulfurs of the ligating cysteines. ${ }^{43}$ Current structural data on the Y. lipolytica or B. taurus enzymes cannot confirm or distinguish either possibility, but a hydrogen bond between the histidine and one of the cysteine ligands was modeled in the structure of (oxidized) T. thermophilus complex I. ${ }^{4,44}$ The observed anisotropy can be satisfactorily reproduced using a homology model for the bovine enzyme created from $T$. thermophilus structural data together with the N2 spin projection factors (describing the magnetic coupling of the four $\mathrm{Fe}$-subsite spins to form an $S=1 / 2$ ground state) we determined previously ${ }^{13}$ (see Supplementary Table 4).

The H1 hydrogen bond to N2 explains the large drop in reduction potential $(76 \mathrm{mV} \text { at } \mathrm{pH} 7)^{24}$ observed in the $\mathrm{H} 226 \mathrm{M}$ variant. Similarly, the reduction potential of the $[3 \mathrm{Fe}-4 \mathrm{~S}]$ cluster in succinate:ubiquinone oxidoreductase dropped by 68 $\mathrm{mV}(\mathrm{pH} 8)$ upon substitution of a second-coordination sphere His thought to be hydrogen-bonded to the cluster with $\mathrm{Thr}^{45}$ Birrell and co-workers ${ }^{46}$ systematically studied the effects of hydrogen bonding on the reduction potential of the all Cysligated $[2 \mathrm{Fe}-2 \mathrm{~S}]$ cluster in HydC from Thermotoga maritima and observed a difference of $58 \mathrm{mV}$ in the reduction potentials of the $\mathrm{V} 131 \mathrm{H}$ and $\mathrm{V} 131 \mathrm{M}$ variants (at $\mathrm{pH} 7$ ). In all cases the hydrogen bond withdraws electron density from the cluster, increasing the stability of the reduced cluster and hence increasing its reduction potential.

The Exchangeable Proton H2. Besides the conserved histidine, there are no other amino acids in the vicinity of $\mathrm{N} 2$ with canonical $\mathrm{p} K_{\mathrm{a}}$ values between 6 and 9. Therefore, $\mathrm{H} 2$ may originate from a bound water molecule located close to N2. Recent molecular dynamics simulations have suggested that many water molecules infiltrate the protein around the quinone-binding channel and the $\mathrm{Fe}-\mathrm{S}$ clusters, ${ }^{47}$ and indeed, they are likely required for proton transfer to and from the histidine. Identification of candidate water molecules may be possible using higher resolution structural data in the future. The substantial anisotropy of $\mathrm{H} 2$ (Table 1) suggests that the $\mathrm{H}_{2} \mathrm{O}$ molecule may be in a preferred orientation with respect to the cluster (albeit free to rotate), but the lack of isotropic interaction and lower magnitude of the anisotropic interaction both imply that it interacts more weakly than H1. It is thus likely that the $\mathrm{pK}$ of $\mathrm{H} 2$ responds only weakly to the cluster oxidation state, such that its $\mathrm{p} K_{\mathrm{ox}}$ and $\mathrm{p} K_{\text {red }}$ are close together, and $\mathrm{H} 1$ dominates the $\mathrm{pH}$ dependence of the $\mathrm{N} 2$ reduction potential (Figure 2).

Effects of His190/226 on Catalysis. To assess the catalytic importance of protonating the conserved histidine, we compared how the activities of wild-type and variant $Y$. lipolytica complex I vary with $\mathrm{pH}$. Using $\mathrm{NADH}$ and decylubiquinone (a soluble analogue of the native ubiquinone), the activity of $\mathrm{H} 226 \mathrm{M}$ was $\sim 80 \%$ that of the wild-type enzyme, and it displayed the same $\mathrm{pH}$-dependent activity with a maximum at $\mathrm{pH} \sim 7.5$ regardless of the presence of the histidine (Supplementary Figure 12). Similarly, in activity assays conducted using ubiquinone-10 in an artificial membrane system comprising complex I and the alternative oxidase to enable $\mathrm{Q}_{10}$ cycling, ${ }^{48}$ the $\mathrm{H} 226 \mathrm{M}$ variant displayed $\sim 65 \%$ of the wild-type activity at $\mathrm{pH}$ 7.5. Previously, it was demonstrated that the $\mathrm{H} 226 \mathrm{M}$ variant is capable of proton pumping as well as of redox catalysis. ${ }^{24}$ Therefore, neither the redox reaction or proton translocation is severely impaired by the absence of His 190/226, and we conclude that proton-coupled electron transfer at cluster $\mathrm{N} 2$ is not required for complex I catalysis.

\section{CONCLUSIONS}

The question whether the terminal Fe-S cluster N2 in complex I is involved in proton pumping was first raised in the 1970s and has remained subject to debate ever since. Here, we have shown that the characteristics of the PCET reaction at N2 are not consistent with it being the coupling reaction that initiates proton translocation. The following lines of evidence led to this conclusion: (1) The limited $\mathrm{pH}$ dependence of the $\mathrm{N} 2$ reduction potential (less than $-60 \mathrm{mV}$ per $\mathrm{pH}$ unit) shows that proton transfer is not strongly coupled to electron transfer. At $\mathrm{pH} 7.4$, the complete reduction of cluster $\mathrm{N} 2$ results in only $50 \%$ of the enzyme molecules taking up a coupled proton, insufficient to support the efficient and stoichiometric proton translocation mechanism that is exhibited by complex I. ${ }^{49}$ Data on the $\mathrm{pH}$ dependence of the $\mathrm{N} 2$ reduction potential are now consistent for complex I from both mammalian and yeast species. (2) Consistent with (1), no strongly coupled exchangeable protons (as would be required for efficient 
energy conversion) could be detected in the vicinity of $\mathrm{N} 2$. Instead, two weakly coupled exchangeable protons are present. The more strongly coupled of these belongs to a conserved histidine in the second coordination sphere of $\mathrm{N} 2$ that we conclude is hydrogen-bonded to the reduced cluster. (3) Despite the profound effect of the conserved histidine residue on the reduction potential and $\mathrm{pH}$ dependence of $\mathrm{N} 2$, enzyme activity is not substantially compromised upon its substitution.

We cannot exclude that protonation at cluster N2 plays an indirect role in complex I catalysis, perhaps by supporting proton uptake for ubiquinone reduction. How protons are supplied to the ubiquinone headgroup is unknown, and clues may result from re-examining the proton environment of the cluster in the presence of a bound substrate analogue or under a membrane potential. The mechanisms of the binding, reduction, and dissociation of ubiquinone/ubiquinol, possibly involving semiquinone ${ }^{3}$ or doubly anionic intermediates, ${ }^{50,51}$ must now be investigated as candidates for the catalytic step that couples proton transfer to electron transfer in this reversible energy-transducing enzyme.

Many essential enzymes such as hydrogenases, nitrogenases, fumarate reductase, carbon-monoxide dehydrogenase, and nitrate reductase contain a multitude of buried iron-sulfur clusters. In this paper, we have pinpointed the properties of a single proton in a giant $1 \mathrm{MDa}$ enzyme that contains a multitude of $\mathrm{Fe}-\mathrm{S}$ clusters, demonstrating how pulse EPR methods can be used to isolate and examine the behavior of single redox-active centers in highly complicated systems.

\section{EXPERIMENTAL SECTION}

Protein Preparations. Bovine complex I was prepared using the protocol of Sharpley et al. ${ }^{52}$ implemented as described previously, ${ }^{32}$ except that the buffer for the Superose 6 Increase column contained 20 $\mathrm{mM}$ Tris, $150 \mathrm{mM} \mathrm{NaCl}, 10 \%(\mathrm{v} / \mathrm{v})$ glycerol, and $0.05 \%(\mathrm{w} / \mathrm{v}) n-$ dodecyl $\beta$-D-maltoside (DDM, Glycon). Complex I from Y. lipolytica was prepared as described previously ${ }^{53}$ except that cells were grown at $30{ }^{\circ} \mathrm{C}$.

Complex I catalytic activities were measured using $200 \mu \mathrm{M}$ NADH and $200 \mu \mathrm{M}$ decylubiquinone in $20 \mathrm{mM}$ Tris- $\mathrm{HCl}, 0.15 \%$ asolectin (Avanti Polar Lipids), 0.15\% 3-[(3-cholamidopropyl)dimethylammonio]-1-propanesulfonate (CHAPS, CalBioChem), $\mathrm{pH}$ 7.5 at $32{ }^{\circ} \mathrm{C}$. Activities for the bovine enzyme were between 10 and 20 $\mu$ mol $\mathrm{NADH} \mathrm{min}^{-1} \mathrm{mg}^{-1}$, and the midpoint potential of $\mathrm{N} 2$ was independent of the activity. The buffer for $\mathrm{pH}$-dependent activity measurements contained $100 \mathrm{mM}$ of each of MES, MOPS, and Tris $\mathrm{pH}$ corrected with $\mathrm{KOH}$ or $\mathrm{H}_{2} \mathrm{SO}_{4}$.

All complex I EPR samples were supplemented with glycerol to a final concentration of $30 \%(\mathrm{v} / \mathrm{v})$.

EPR Sample Buffers. Temperature-independent $\mathrm{pH}$ buffers were used to set $\mathrm{pH}$ values between 6.0 and 9.0 (see Supporting Information text and Supplementary Table 1 for details). The buffer used to obtain the pH 5.0 data point in Figure 1 (see also Supplementary Figure 1) contained $100 \mathrm{mM}$ citric acid, $150 \mathrm{mM}$ $\mathrm{NaCl}$, and $30 \%(\mathrm{v} / \mathrm{v})$ glycerol and was adjusted to $\mathrm{pH} 6.0$ at room temperature. Using $\mathrm{pH}$ indicators (see Supporting Information), we established that its $\mathrm{pH}$ drops by $1.0 \pm 0.2$ units from 298 to $77 \mathrm{~K}$. The $\mathrm{pH} 7.4$ data point in Figure 2 was prepared in $100 \mathrm{mM}$ MES, $150 \mathrm{mM}$ $\mathrm{NaCl}$, and $30 \%(\mathrm{v} / \mathrm{v})$ glycerol and adjusted to $\mathrm{pH} 6.0$ at room temperature to give a final $\mathrm{pH}$ of $7.4 \pm 0.2$ at $77 \mathrm{~K}$.

Preparation of EPR Samples. Prior to all titrations, bovine complex I was exchanged into the desired buffer and $\mathrm{pH}$ using an Amicon Ultra 0.5 centrifugal filter with an Ultracel-100 membrane (Vivaspin). The protein concentration was typically $10 \mu \mathrm{M}$ except for two titrations at $\mathrm{pH} 6.0$ and 9.0 at $30 \mu \mathrm{M}$. Reduction potential titrations were carried out as described previously. ${ }^{32}$ Briefly, $140 \mu \mathrm{L}$ of sample was stirred in an electrochemical cell inside an anaerobic glovebox (Braun UniLab-plus, $\mathrm{O}_{2}<0.5 \mathrm{ppm}$ ) for $10 \mathrm{~min}$ at $6{ }^{\circ} \mathrm{C}$ to remove $\mathrm{O}_{2}$. Redox mediators (anthraquinone-2-sulfonate, benzyl viologen, indigodisulfonate, indigotrisulfonate, and methylene blue) were each added to the same final molar concentration as the protein. Then, the reduction potential of the solution $\left(\right.$ at $6{ }^{\circ} \mathrm{C}$ ) was set by successive substoichiometric submicroliter additions of sodium dithionite (in $10 \mathrm{mM}$ Tris buffer, $\mathrm{pH} 8$ ) and monitored by opencircuit potentiometry using an $\mathrm{EmStat}^{+}$(PalmSens). Once the desired potential was attained, $9 \mu \mathrm{L}$ of solution was transferred to an EPR tube, flash-frozen inside the glovebox, and stored in liquid nitrogen. Pulse EPR measurements (Figures 3 and 4) were conducted on samples selected from those described above on the basis of the maximum reduction of $\mathrm{N} 2$ with all other EPR-visible clusters fully oxidized $(-167 \mathrm{mV}$ at $\mathrm{pH}$ 6.0, see Supplementary Figure 1A, and $-145 \mathrm{mV}$ at $\mathrm{pH}$ 9.0). The bovine $\mathrm{pD} 6.0$ sample (protein concentration $30 \mu \mathrm{M},-160 \mathrm{mV}$ ) was made using the same procedure as for a full titration except that $\mathrm{D}_{2} \mathrm{O}$ and deuterated glycerol- $d_{3}$ (Sigma-Aldrich) were used. The $\mathrm{pD}$ was adjusted using $\mathrm{pD}=\mathrm{pH}$ meter reading +0.41 .

$\mathrm{NADH}$-reduced samples of native and $\mathrm{H} 226 \mathrm{M}$ Y. lipolytica complex I (final concentration $30 \mu \mathrm{M}$ ) were prepared in the glovebox by the addition of $1 \mu \mathrm{L}$ of $40-50 \mathrm{mM}$ NADH to $15 \mu \mathrm{L}$ of complex I solution at $\mathrm{pH} 6.5$ or $\mathrm{pD} 6.5$ (see Supplementary Table 1 for buffer composition). The $\mathrm{pH}$ of 6.5 is slightly higher than for the equivalent bovine complex I samples due to the instability of concentrated $Y$. lipolytica complex I at $\mathrm{pH} 6.0$, but His 226 is still $92 \%$ protonated. Solutions were transferred to EPR tubes and flash-frozen inside the glovebox.

All reduction potentials are reported relative to the potential of the standard hydrogen electrode (SHE). The offset of the reference electrode (DRI-REF-2, World Precision Instruments), $+200 \mathrm{mV}$, was determined using quinhydrone as a standard.

EPR Spectroscopy. EPR measurements were performed using an X/Q-band Bruker Elexsys E500 spectrometer equipped with a closedcycle cryostat (Cryogenic Ltd., UK). All X-band measurements were carried out in an ER 4118X-MS2 resonator (Bruker), operated in either continuous wave $(\mathrm{CW})$ or pulse mode. $\mathrm{CW}$ spectra were baseline subtracted using a buffer-only sample measured under the same conditions. CW measurements of titration samples were carried out at $25 \mathrm{~K}, 2 \mathrm{~mW}$ microwave power, modulation amplitude $0.7 \mathrm{mT}$ at $100 \mathrm{kHz}$ modulation frequency.

Two-pulse echo-detected field sweeps (EDFS) were acquired with the pulse sequence $\pi / 2-\tau-\pi-\tau-$ echo $(\pi / 2=16 \mathrm{~ns}, \pi=32$, and $\tau=$ $200 \mathrm{~ns})$. HYSCORE spectra were recorded with the 4-pulse sequence $\pi / 2-\tau-\pi / 2-t 1-\pi-t 2-\pi / 2-\tau-$ echo; $\pi / 2=16 \mathrm{~ns}, \pi=16 \mathrm{~ns}$ at twice the power of the $\pi / 2$ pulses (i.e., in a second channel), $t_{1}=t_{2}=80 \mathrm{~ns}$, $\tau$ given in figure legends, 4-step phase cycle $[0,0,0,0]-\left[0,0,0,180^{\circ}\right]+$ $\left[0,0,180^{\circ}, 0\right]-\left[0,0,180^{\circ}, 180^{\circ}\right]$. SMART HYSCORE spectra were recorded with the 4-pulse sequence HTA-t1- $\pi-t 2-\mathrm{HTA}-\tau-\pi-\tau-$ echo (4-step phase cycle $[0,0,0,0]-\left[0,0,180^{\circ}, 0\right]+\left[0,0,0,180^{\circ}\right]-$ $\left.\left[0,0,180^{\circ}, 180^{\circ}\right]\right)$ with $\pi=32 \mathrm{~ns}$, high turning angle (HTA) pulse $=40$ $\mathrm{ns}$, and $t_{1}=t_{2}=96 \mathrm{~ns}$. Because of the spectral asymmetry about the diagonal (typical for SMART-HYSCORE spectra), the SMART HSYCORE spectra were symmetrized. ${ }^{54}$

REFINE experiments were carried out with a $T_{1}$ filter $\left(T_{\mathrm{f}}\right)$ of $62 \mu \mathrm{s}$ and $\pi=32 \mathrm{~ns}$ initial inversion pulse prior to the EDFS pulse sequence $\left(\pi-T_{\mathrm{f}}-\pi / 2-\tau-\pi-\tau-\right.$ echo, single channel $)$ or HYSCORE pulse sequence $\left(\pi-T_{\mathrm{f}}-\pi / 2-\tau-\pi / 2-\mathrm{t} 1-\pi-t 2-\pi / 2-\tau-\right.$ echo $)$, where $t_{1}=$ $t_{2}=80 \mathrm{~ns}$ and $\tau$ is specified in the figure legends. As for standard HYSCORE experiments, the fourth pulse in REFINE HYSCORE experiments $(\pi=16 \mathrm{~ns})$ was placed in a different channel to all other pulses. Pulse EPR measurements of bovine complex I samples (with $\mathrm{N} 2$ as the sole paramagnetic species) were carried out at $10 \mathrm{~K}$ to maximize the signal-to-noise ratio. Pulse EPR experiments of NADHreduced $Y$. lipolytica complex I were carried out at $14 \mathrm{~K}$ to eliminate contributions from N3 and N4 (N1b could be filtered out using the relaxation filter). The HYSCORE spectra obtained from Y. lipolytica were symmetrized $^{55,56}$ (Figure 5) due to their lower signal-to-noise compared to their bovine counterparts measured at $10 \mathrm{~K}$. Additional (variable) experimental parameters are provided in the figure legends. 
Typical acquisition times were $14 \mathrm{~h}$ for HYSCORE, $24 \mathrm{~h}$ for REFINE HYSCORE, and $60 \mathrm{~h}$ for SMART HYSCORE experiments.

Analyses of EPR Data. The reduction potentials of N2 at each $\mathrm{pH}$ were determined by plotting the integrals of the simulated N2 spectra as a function of potential and by fitting the resulting data to the oneelectron Nernst equation (see Supplementary Figure 1 and ref 32). The data points shown in Figure 2 were fitted with the equation

$$
E_{\mathrm{m}}\left(\left[H^{+}\right]\right)=E_{\mathrm{alk}}+\frac{2.3 R T}{n F} \log _{10}\left(\frac{1+\frac{\left[H^{+}\right]}{K_{\mathrm{red}}}}{1+\frac{\left[H^{+}\right]}{K_{\mathrm{ox}}}}\right)
$$

where $T=282 \mathrm{~K}$ (temperature at which the titrations were carried out), $n=1, E_{\mathrm{alk}}=-175 \mathrm{mV}, K_{\mathrm{ox}}=10^{-5.6}$, and $K_{\mathrm{red}}=10^{-7.4}$. Errors in the values were estimated from the error in the $\mathrm{pH}$ value of the buffer at cryogenic temperatures $( \pm 0.2 \mathrm{pH}$ units $)$ and the error in the fit of the experimental data to the Nernst equation $( \pm 10 \mathrm{mV})$.

HYSCORE difference spectra (Figures $3 \mathrm{~A}$ and $\mathrm{B}$ and 5) were obtained by normalizing the nitrogen cross peaks in the $(-,+)$ quadrant (Supplementary Figure 4) to the same intensity using spectra recorded under the same experimental conditions (microwave frequency, magnetic field position, and $\tau$ ). To obtain the hyperfine coupling parameters of the exchangeable proton couplings revealed by the subtractions, we performed contour line shape analysis (a plot of $\nu_{\alpha}^{2}$ versus $\nu_{\beta}^{2}$, Supplementary Figure 8) of the cross peaks as a first approximation and as previously described. ${ }^{36}$ The hyperfine coupling parameters of $\mathrm{H} 1$ and $\mathrm{H} 2$ (Supplementary Table 2) were then further refined through simulations of all pulse EPR data as described in the Supporting Information.

\section{ASSOCIATED CONTENT}

\section{(S Supporting Information}

The Supporting Information is available free of charge on the ACS Publications website at DOI: 10.1021/jacs.7b09261.

Potentiometric titrations of bovine complex I; design, composition, and verification of temperature-independent $\mathrm{pH}$ buffer systems; HYSCORE analysis and simulation procedures; and estimation of hyperfine coupling anisotropy of $\mathrm{H} 1$ based on structural information (PDF)

\section{AUTHOR INFORMATION}

\section{Corresponding Author}

*m.roessler@qmul.ac.uk

\section{ORCID 우}

Judy Hirst: 0000-0001-8667-6797

Maxie M. Roessler: 0000-0002-5291-4328

Notes

The authors declare no competing financial interest.

\section{ACKNOWLEDGMENTS}

We thank Prof. Conrad Mullineaux (QMUL) for use of his fluorescence spectrometer and Camille Galateau (QMUL) for carrying out some of the $\mathrm{pH}$ indicator and fluorescence measurements. Prof. Ulrich Brandt (Radboud Center for Mitochondrial Medicine, The Netherlands) is gratefully acknowledged for providing the Y. lipolytica H226M strain. We are grateful to Prof. Jeffrey Harmer (The University of Queensland) and to the reviewers for helpful comments on the manuscript. This research was funded by the EPSRC (First Grant EP/M024393/1 to M.M.R. and studentship EP/ M506394/1 to J.J.W.) and by The Medical Research Council (U105663141 to J.H.).

\section{REFERENCES}

(1) Fassone, E.; Rahman, S. J. Med. Genet. 2012, 49, 578.

(2) Vafai, S. B.; Mootha, V. K. Nature 2012, 491, 374.

(3) Hirst, J.; Roessler, M. M. Biochim. Biophys. Acta, Bioenerg. 2016, 1857, 872.

(4) Baradaran, R.; Berrisford, J. M.; Minhas, G. S.; Sazanov, L. A. Nature 2013, 494, 443.

(5) Vinothkumar, K. R.; Zhu, J.; Hirst, J. Nature 2014, 515, 80.

(6) Zhu, J.; Vinothkumar, K. R; Hirst, J. Nature 2016, 536, 354.

(7) Zickermann, V.; Wirth, C.; Nasiri, H.; Siegmund, K.; Schwalbe, H.; Hunte, C.; Brandt, U. Science 2015, 347, 44.

(8) Fiedorczuk, K.; Letts, J. A.; Degliesposti, G.; Kaszuba, K.; Skehel, M.; Sazanov, L. A. Nature 2016, 538, 406.

(9) Volbeda, A.; Charon, M.-H.; Piras, C.; Hatchikian, E. C.; Frey, M.; Fontecilla-Camps, J. C. Nature 1995, 373, 580.

(10) Lancaster, C. R. D.; Michel, H.; Kröger, A.; Auer, M. Nature 1999, 402, 377.

(11) Dobbek, H.; Svetlitchnyi, V.; Gremer, L.; Huber, R.; Meyer, O. Science 2001, 293, 1281.

(12) Bertero, M. G.; Rothery, R. A.; Palak, M.; Hou, C.; Lim, D.; Blasco, F.; Weiner, J. H.; Strynadka, N. C. J. Nat. Struct. Biol. 2003, 10, 681.

(13) Roessler, M. M.; King, M. S.; Robinson, A. J.; Armstrong, F. A.; Harmer, J.; Hirst, J. Proc. Natl. Acad. Sci. U. S. A. 2010, 107, 1930.

(14) Verkhovskaya, M. L.; Belevich, N.; Euro, L.; Wikstrom, M.; Verkhovsky, M. I. Proc. Natl. Acad. Sci. U. S. A. 2008, 105, 3763.

(15) de Vries, S.; Doerner, K.; Strampraad, M. J. F.; Friedrich, T. Angew. Chem., Int. Ed. 2015, 54, 2844.

(16) Hexter, S. V.; Grey, F.; Happe, T.; Climent, V.; Armstrong, F. A. Proc. Natl. Acad. Sci. U. S. A. 2012, 109, 11516.

(17) Adamson, H.; Robinson, M.; Wright, J. J.; Flanagan, L. A.; Walton, J.; Elton, D.; Gavaghan, D. J.; Bond, A. M.; Roessler, M. M.; Parkin, A. J. Am. Chem. Soc. 2017, 139, 10677.

(18) Lubitz, W.; Ogata, H.; Rüdiger, O.; Reijerse, E. Chem. Rev. 2014, $114,4081$.

(19) Armstrong, F. A.; Evans, R. M.; Hexter, S. V.; Murphy, B. J.; Roessler, M. M.; Wulff, P. Acc. Chem. Res. 2016, 49, 884.

(20) Brandt, U. Annu. Rev. Biochem. 2006, 75, 69.

(21) Gutman, M.; Singer, T. P.; Beinert, H. Biochemistry 1972, 11, 556.

(22) Ingledew, W. J.; Ohnishi, T. Biochem. J. 1980, 186, 111.

(23) Yano, T.; Dunham, W. R.; Ohnishi, T. Biochemistry 2005, 44, 1744.

(24) Zwicker, K.; Galkin, A.; Dröse, S.; Grgic, L.; Kerscher, S.; Brandt, U. J. Biol. Chem. 2006, 281, 23013.

(25) Meinhardt, S. W.; Wang, D. C.; Hon-nami, K.; Yagi, T.; Oshima, T.; Ohnishi, T. J. Biol. Chem. 1990, 265, 1360.

(26) Leif, H.; Sled, V. D.; Ohnishi, T.; Weiss, H.; Friedrich, T. Eur. J. Biochem. 1995, 230, 538.

(27) Garofano, A.; Zwicker, K.; Kerscher, S.; Okun, P.; Brandt, U. J. Biol. Chem. 2003, 278, 42435.

(28) Yakovlev, G.; Reda, T.; Hirst, J. Proc. Natl. Acad. Sci. U. S. A. 2007, 104, 12720.

(29) Birrell, J. A.; Morina, K.; Bridges, H. R.; Friedrich, T.; Hirst, J. Biochem. J. 2013, 456, 139.

(30) Maly, T.; MacMillan, F.; Zwicker, K.; Kashani-Poor, N.; Brandt, U.; Prisner, T. F. Biochemistry 2004, 43, 3969.

(31) Maly, T.; Zwicker, K.; Cernescu, A.; Brandt, U.; Prisner, T. F. Biochim. Biophys. Acta, Bioenerg. 2009, 1787, 584.

(32) Wright, J. J.; Salvadori, E.; Bridges, H. R.; Hirst, J.; Roessler, M. M. J. Inorg. Biochem. 2016, 162, 201.

(33) Williams-Smith, D. L.; Bray, R. C.; Barber, M. J.; Tsopanakis, A. D.; Vincent, S. P. Biochem. J. 1977, 167, 593.

(34) Moore, G. R.; Williams, R. J.; Peterson, J.; Thomson, A. J.; Mathews, F. S. Biochim. Biophys. Acta, Protein Struct. Mol. Enzymol. 1985, 829, 83.

(35) Lhee, S.; Kolling, D. R. J.; Nair, S. K.; Dikanov, S. A.; Crofts, A. R. J. Biol. Chem. 2010, 285, 9233. 
(36) Kolling, D. R. J.; Samoilova, R. I.; Shubin, A. A.; Crofts, A. R.; Dikanov, S. A. J. Phys. Chem. A 2009, 113, 653.

(37) Dikanov, S. A.; Bowman, M. K. J. Magn. Reson., Ser. A 1995, 116, 125 .

(38) Flores, M.; Isaacson, R.; Abresch, E.; Calvo, R.; Lubitz, W.; Feher, G. Biophys. J. 2007, 92, 671.

(39) Grimaldi, S.; Arias-Cartin, R.; Lanciano, P.; Lyubenova, S.; Szenes, R.; Endeward, B.; Prisner, T. F.; Guigliarelli, B.; Magalon, A. J. Biol. Chem. 2012, 287, 4662.

(40) Dikanov, S. A.; Samoilova, R. I.; Kolling, D. R. J.; Holland, J. T.; Crofts, A. R. J. Biol. Chem. 2004, 279, 15814.

(41) Liesum, L.; Schweiger, A. J. Chem. Phys. 2001, 114, 9478.

(42) Noodleman, L. Nat. Chem. Biol. 2016, 12, 391.

(43) Hirano, Y.; Takeda, K.; Miki, K. Nature 2016, 534, 281.

(44) Sazanov, L. A.; Hinchliffe, P. Science 2006, 311, 1430.

(45) Ruprecht, J.; Iwata, S.; Rothery, R. A.; Weiner, J. H.; Maklashina, E.; Cecchini, G. J. Biol. Chem. 2011, 286, 12756.

(46) Birrell, J. A.; Laurich, C.; Reijerse, E. J.; Ogata, H.; Lubitz, W. Biochemistry 2016, 55, 4344.

(47) Di Luca, A.; Gamiz-Hernandez, A. P.; Kaila, V. R. I. Proc. Natl. Acad. Sci. U. S. A. 2017, 114, E6314.

(48) Jones, A. J. Y.; Blaza, J. N.; Bridges, H. R.; May, B.; Moore, A. L.; Hirst, J. Angew. Chem., Int. Ed. 2016, 55, 728.

(49) Pryde, K. R.; Hirst, J. J. Biol. Chem. 2011, 286, 18056.

(50) Brandt, U. Biochim. Biophys. Acta, Bioenerg. 2011, 1807, 1364.

(51) Efremov, R. G.; Sazanov, L. A. Biochim. Biophys. Acta, Bioenerg. 2012, 1817, 1785.

(52) Sharpley, M. S.; Shannon, R. J.; Draghi, F.; Hirst, J. Biochemistry 2006, 45, 241.

(53) Varghese, F.; Atcheson, E.; Bridges, H. R.; Hirst, J. Hum. Mol. Genet. 2015, 24, 6350.

(54) De Cooman, H.; Pauwels, E.; Vrielinck, H.; Sagstuen, E.; Van Doorslaer, S.; Callens, F.; Waroquier, M.; Callens, F. Phys. Chem. Chem. Phys. 2009, 11, 1105.

(55) Klein, E. L.; Astashkin, A. V.; Raitsimring, A. M.; Enemark, J. H. Coord. Chem. Rev. 2013, 257, 110.

(56) Albertini, M.; Galazzo, L.; Maso, L.; Vallese, F.; Berto, P.; De Rosa, E.; Di Valentin, M.; Costantini, P.; Carbonera, D. Top. Catal. 2015, 58, 708. 